\title{
The Visualization Analysis of Research Hotspot and Frontier Technology of the Smart Power Distribution and Utilization Based on the Cite Space
}

\author{
Tang-Yun Xu' ${ }^{1}$, Hai-Ni Qu${ }^{1}$, San-Shan Zhao', Mei-Xia Zhang², Xiu Yang'ㄹ, Xu-Yan Huang² \\ ${ }^{1}$ Electric Power Research Institute, State Grid Shanghai Municipal Electric Power Company, Shanghai, China \\ ${ }^{2}$ School of Electrical Engineering, Shanghai University of Electric Power, Shanghai, China \\ Email: hxyshiep@163.com
}

How to cite this paper: $\mathrm{Xu}, \mathrm{T} .-\mathrm{Y}$., $\mathrm{Qu}$, H.-N., Zhao, S.-S., Zhang, M.-X., Yang, X. and Huang, X.-Y. (2017) The Visualization Analysis of Research Hotspot and Frontier Technology of the Smart Power Distribution and Utilization Based on the Cite Space. Energy and Power Engineering, 9, 515-524. https://doi.org/10.4236/epe.2017.94B057

Received: January 15, 2017

Accepted: March 30, 2017

Published: April 6, 2017

\begin{abstract}
In order to make an intensive study of the development of smart power distribution and utilization technology in China, their research hotspots and frontier technology are selected out through combining the informatics method, and using the CiteSpace which can take keyword co-occurrence analysis and draw the visualization graph. According to this result, we can infer the development trend of smart power distribution and utilization in the future, and providing reference for the researcher whose engage in this domain. The electric related literature was collected from the CNKI database in China. Under the smart power distribution and utilization domain, we also analyze the development of the power quality and the energy internet in detail.
\end{abstract}

\section{Keywords}

Smart Power Distribution and Utilization, Informetrics, CiteSpace, Visualization Graph

\section{Introduction}

In recent years, the development of the power distribution and utilization has made remarkable achievements in China. During the period of "12th Five-Year", we had scored remarkable achievements in the areas of power supply capacity, quality of power supply and utilization. Looking forward to "13th Five-Year", the power distribution and utilization technology will tend to be great of intelligence, informatization and automation [1].

Some scholars have analyzed the relevant technologies in the field of power 
distribution and utilization. Literature [2] introduced the intelligent process of electricity construction and development in China from the intelligent power distribution network optimal planning, self-healing structure design, and intelligent with electric measurement and communication technology. Since more and more distributed energy, energy storage device and electric vehicle are connected to the power grid, the stability of the power grid will be subject to certain challenges. Literature [3] discussed the intelligent power distribution system architecture of distributed energy with high permeability, and discussed the prospect of the intelligent power distribution system from two angles: energy internet and integrated energy system; It analyzed intelligent power utilization technology and mechanism, and put forward the direction for the future of smart power as well as challenges from the perspective of promoting renewable energy given [4].

In the future, mastering the trend of the electric technology development and research emphasis becomes very important. Literature [5] proposed the future development trend of the power distribution and utilization technique, from the active power distribution system technology and energy internet. In the future, we will achieve intelligent management with power distribution and utilization, the large data technology will play an important role in this operating process, under the big data environment, [6] proposed the research thought and the method from the two typical application scenes, user electricity behavior and the load forecast. To improve the electrical automation and power grid intelligence, the technical staff will collect, process and real-time monitor the user's electricity consumption information, through electrical system integration technology system which takes AMI as the core [7].

In this paper, we adopt analysis methods based on informetrics, applying those methods into the analysis, and summarizing the research hotspots and cutting-edge technology in the field of the smart power distribution and utilization by the visualization software CiteSpace, which can draw out the visualization graph, and dispose the related literatures by keyword and cluster analysis.

\section{Informetrics and Its Analysis Software}

Informetrics derives from scientometrics and literature metrology [8]. It takes intelligence (data, text, literature, etc.) as the research object, by the methods of statistics and the citation analysis, so as to reveal the structure and regularity of the intelligence. In era of big data, for knowledge in the field of research, based on the relevant theory and research methods of information metrology, we can analyze the literature of network data in the database and the depth of mining, and find out the correlation between knowledge and exploring the development of the field in the complex data. The literature [9] expounds the dynamic development of informetrics, outlines the knowledge structure of the metrology literature in the intelligence based on the statistical analysis of the literature from 2001 to 2010. In [10], on the basis of the informatics theory, from the aspects of research productive forces, research influence, research innovation power and 
research development strength, it analyzed the international influence of the Chinese "985" project universities.

At present, the informetrics research method contains statistical publications, author statistics, citation analysis, frequency analysis, content analysis, and index analysis and so on, and recent methods commonly used have co-citation analysis, co-occurrence analysis, factor analysis, multidimensional scaling analysis, cluster analysis, spectrum analysis. For different data type, the existing methods can deal with the structured data and limited number of keywords by cluster analysis, co-occurrence analysis, etc. However, when disposing the unstructured data and semi-structured data, as well as a greater number of data resource, they shows some limitation [11].

The main research tools of the informetrics include SPSS, Ucinet, SAS and CiteSpace. The CiteSpace as visualization software to measure and analyze of scientific literature data information, compared with other softwares, it's relatively perfect in functions, particularly in terms of time series analysis and emergency monitoring. In 2004, Chen and his team used Java language to develop CiteSpace, the professor of the college of information science and technology, University of Drexel in Philadelphia, PA, USA. There has some certain practice at home and abroad for the CiteSpace. The main application fields focus on the library and archives management, and management science and engineering and pedagogy [12]. It illustrates the function of methodology of CiteSpace Knowledge Graph from the four aspects about core function, theory, application process and to expand functions in [13]. We can use CiteSpace to infer the current research focus and developing direction of Intelligent Tourism in China [14].

In this paper, we will combine with the analysis of co-occurrence information metrology and cluster analysis methods, using the CiteSpace software to take the visualization analysis for the power distribution and utilization related literatures downloaded from CNKI database.

\section{CiteSpace Operations}

The analysis of the smart power distribution and utilization technology is mainly from the following three steps:

1) The data collection. CiteSpace can deal with the literatures from the WoS, ADC, CNKI, CSSCI databases. Besides the WoS and arXiv, other databases need to take a data preprocessing, the downloaded literature will be converted to WoS format. In order to study the development of the smart power distribution and utilization key technology in China, this paper collected data from the CNKI database.

2) The selection of analyses object. The transformed data were done visualization view analysis in CiteSpace, analysis objects are divided into citing articles and cited reference, citing articles could be done the co-occurrence analysis, it contains the information of the author, organization, country, title, keywords, etc. The cited reference could be done the co-citation analysis. The choice of ob- 
ject determines the node type, also determines the CiteSpace analysis purposes.

3) Visualization analysis. Visualization graph contain cluster, time-zone and timeline view in CiteSpace, the default view is cluster view. Three kinds of view are different on the description of the node. The cluster view focuses on the knowledge structure of the different research fields; time-zone view depicts the evolution of the research topics with time trends and influence; timeline view describes the relationship between clusters and the history of literature in a cluster span [15].

Specific analysis flow chart is shown in Figure 1.

\section{The Key Technology}

To analyze the key technologies of the smart power distribution and utilization, firstly, we select eight higher-ranked Chinese journals in the electric power industry, including academic journals and engineering journals. They are "Proceedings of The CSEE", "Power System Technology", "Automation of Electric Power Systems", "Electric Power Automation Equipment", and other four non-EI journals. Then, downloading their all literatures from 2000 to 2016 in CNKI database; secondly, after the data preprocessing, taking the keywords co-occurrence analysis for each journal; finally, screening the high frequency keywords about smart power distribution and utilization. Through the comparison and analysis, we inferred several key research hotspots of the current study; Table 1 and Table 2 list the four EI journals and their high frequency keywords.

According to these two tables, we found the frequency of the power quality, reactive power optimization, power supply reliability, distributed power supply, relay protection, demand response, electric cars are higher than others, and their rankings also are more prominent in the four EI journals. Consequently, we can speculate that they are the research hotspots in smart power distribution and utilization domain. In recent years, the active distribution network and the

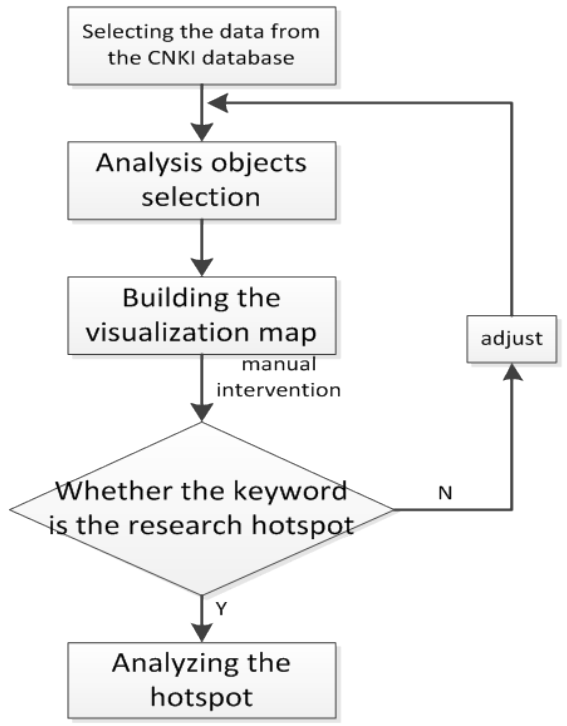

Figure 1. Operation process diagram. 
Table 1. Keywords in "Proceedings of the CSEE" and "Automation of electric power systems".

\begin{tabular}{ccccc}
\hline & \multicolumn{2}{c}{ Proceedings of The CSEE } & \multicolumn{2}{c}{$\begin{array}{c}\text { Automation of Electric } \\
\text { Power Systems }\end{array}$} \\
\cline { 2 - 5 } & keyword & Freq & keyword & Freq \\
\hline 1 & power quality & 87 & distributed power & 149 \\
2 & energy internet & 55 & power quality & 122 \\
3 & distributed power & 48 & reliability & 112 \\
4 & micro-grid & 47 & electric vehicle & 90 \\
& electric vehicle & 45 & distribution & 80 \\
5 & harmonic analysis & 45 & automation & \\
& & & power system & 75 \\
6 & reactive compensation & 38 & reliability assessment & 55 \\
7 & reliability analysis & 35 & distributed generation & 53 \\
9 & reactive-power & 34 & demand response & 37 \\
& optimization & & reactive-power & 31 \\
\hline & load prediction & 26 & optimization & \\
\hline
\end{tabular}

Table 2. Keywords in "Power system technology" and "Electric power automation equipment".

\begin{tabular}{ccccc}
\hline \multirow{2}{*}{ No } & \multicolumn{2}{c}{ Power System Technology } & \multicolumn{2}{c}{$\begin{array}{c}\text { Electric Power Automation } \\
\text { Equipment }\end{array}$} \\
\cline { 2 - 5 } & keyword & Freq & keyword & Freq \\
\hline 1 & power quality & 156 & protective relaying & 183 \\
2 & reliability & 92 & power quality & 142 \\
3 & distributed power & 85 & harmonic analysis & 105 \\
& reactive-power & & & \\
4 & optimization & 80 & reliability & 99 \\
& voltage stability & 69 & micro-grid & 69 \\
6 & reactive compensation & 68 & reactive compensation & 63 \\
7 & Power system state & 54 & distributed power & 48 \\
& estimation & & electric vehicle & 46 \\
8 & reliability assessment & 53 & distributed generation & 44 \\
9 & distributed generation & 48 & sensitivity analysis & 41 \\
10 & electric vehicle & 48 & reactive power & 32 \\
11 & grid planning & 41 & power-factor & 32 \\
12 & Demand response & 31 & correction & \\
\hline
\end{tabular}

energy internet appear in 2013 and 2010 respectively for the first time. However, their frequencies are not many; they represent the development tendency of the power distribution and utilization technology, even the power industry research direction. Therefore, in the analysis of frontier technology, they should be contained in the scope of the study.

\section{Research Hotspot and Frontier Technology}

After screening hotspots through eight core journals, to explore the evolution of 
hotspots and frontier technology in smart power distribution and utilization, we need to do further analysis. Next, the power quality and the energy interne would be analyzed.

\subsection{Power Quality}

1) After determining the power quality as a research hot spot, we selection the related with the power quality of power distribution and utilization as a standard for document filtering. In CNKI retrieval interface, selecting "power quality" as the theme, "power distribution", "power utilization", or "power distribution and utilization" as keywords for literature retrieval, we found 465 articles, the literatures be taken cluster analysis in CiteSpace.

Node information about power quality as shown in Table 3, lists the keywords appear frequency, betweeness centrality (reflect an indicator of the importance of nodes in the network structure, using this to identify and measure the importance of literature), and the first appear year. Power quality keyword is mainly concentrated in the node which has higher frequency and the betweeness centricity, including the distributed power, reactive power compensation, voltage fluctuations/fall/sag, power factor, reactive power optimization, static reactive power compensator.

2) Timeline view

Figure 2 is the timeline view about the power quality, the same clustering's literatures are placed in the same level. At the top of the view, it is year's color bar, from blue to orange-red gradient, the more to the right, the later years. The left side of the figure is the timeline, their length is not the same, showing the time span of each cluster, and each timeline shows some key nodes concerned.

The keyword in the Timeline, for example, the first timeline corresponds to the cluster 0, the time span from 2000 to 2016. In this line, According to the emerging year of the keyword, listing "power quality" and "harmonic current" and "distribution transformer" from left to right. Keywords font size indicates how much frequency of their occurrence; the higher the frequency, the bigger

Table 3. Table type styles (Table caption is indispensable).

\begin{tabular}{ccccc}
\hline \multirow{2}{*}{ No } & \multicolumn{4}{c}{ Table Column Head } \\
\cline { 2 - 5 } & Freq & Centrality & Notes & Appear year \\
\hline 1 & 225 & 0.97 & power quality & 2000 \\
2 & 34 & 0.06 & distributed power & 2007 \\
3 & 24 & 0.06 & power distribution & 2000 \\
4 & 21 & & system & 2003 \\
5 & 17 & 0.04 & reactive compensation & 2003 \\
6 & 13 & 0.05 & distributed generation & 2009 \\
7 & 13 & 0.03 & smart grid & 2003 \\
9 & 13 & 0.02 & voltage drop & 2004 \\
10 & 12 & 0.02 & voltage fluctuation & 2006 \\
& 9 & 0.02 & voltage sag & 2004 \\
\hline
\end{tabular}




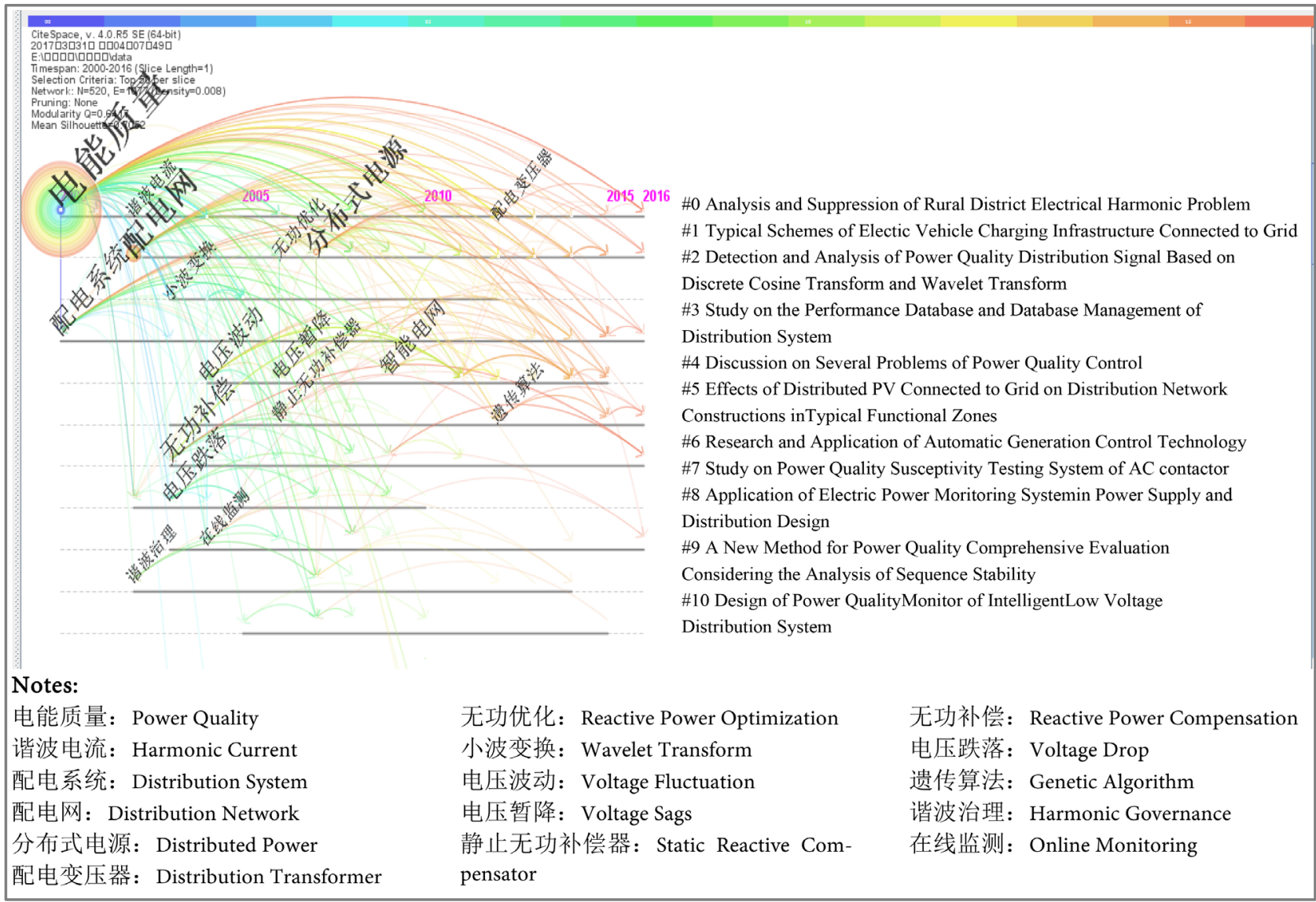

Figure 2. The timeline view of the power quality.

the font size, we can adjust the number of keywords appeared in the time line by changing the threshold.

Cluster name. The red font is the name of the cluster on the Figure 2, the time line is the use of "title terms" to name the cluster (name tag come from the clustering of sorting the highest literature title).Cluster 0 "rural power distribution network analysis and the harmonic suppression" derived from the literature the "rural power distribution network analysis and the harmonic suppression", which is the highest reference in the cluster 0 .

The analysis of the branch technology of the power quality, based on the cluster of information spread and the application documents in the cluster 0 , we deduced their main focuses are harmonic analysis and the technology of distribution network. Similarly, Figure 2 vividly shows 11 clusters, we collected the data of each cluster, including the literature data, then, summing up the following several key technologies about power quality:

1) Harmonic Analysis and Suppression Techniques

2) Distributed grid-connected and monitoring technology

3) Signal detection and evaluation techniques;

4) Reactive power compensation technology;

5) Voltage drop amplitude assessment and fault location technology;

6) Power electronics technology. 


\subsection{Energy Internet}

We conducted keywords co-occurrence analysis about the research of the energy internet. Figure 3 shows the keywords co-occurrence network of high frequency keywords in energy internet. By the same token, the frequency of the keyword appears much determines the circle size; the line between keywords expressed their close degree. The color of the line indicates the corresponding time slices, such as, the blue line between the energy internet and the distributed energy shows their first co-occurrence relation occurred in 2010.

On the base of Figure 3, we found that "energy internet", "smart grid", "distributed energy", "big data", "information on physical system" are top core keywords in energy internet. According to the analysis steps about the power quality before, combining with the literature data and taking cluster analysis, we can find that the current energy internet technology research mainly concentrated in the following aspects:

1) Internet system architecture design and optimization of energy;

2) Physical/information communication technology;

3) New energy application technology;

4) The power energy storage technology;

5) Power distribution system planning technology of the Internet of energy;

6) Energy router;

7) Big data technology.

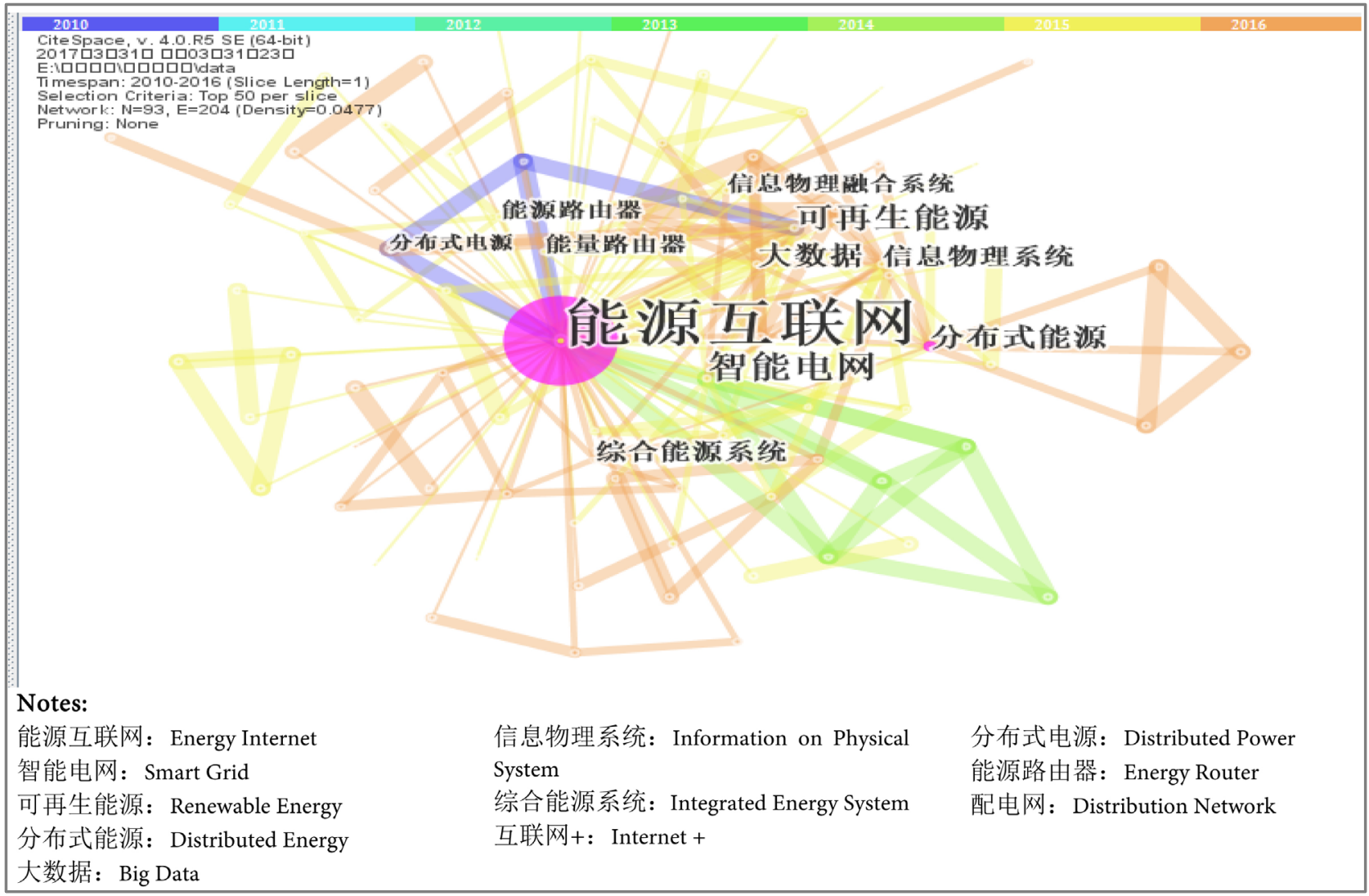

Figure 3. The keyword co-occurrence network view of the energy internet. 


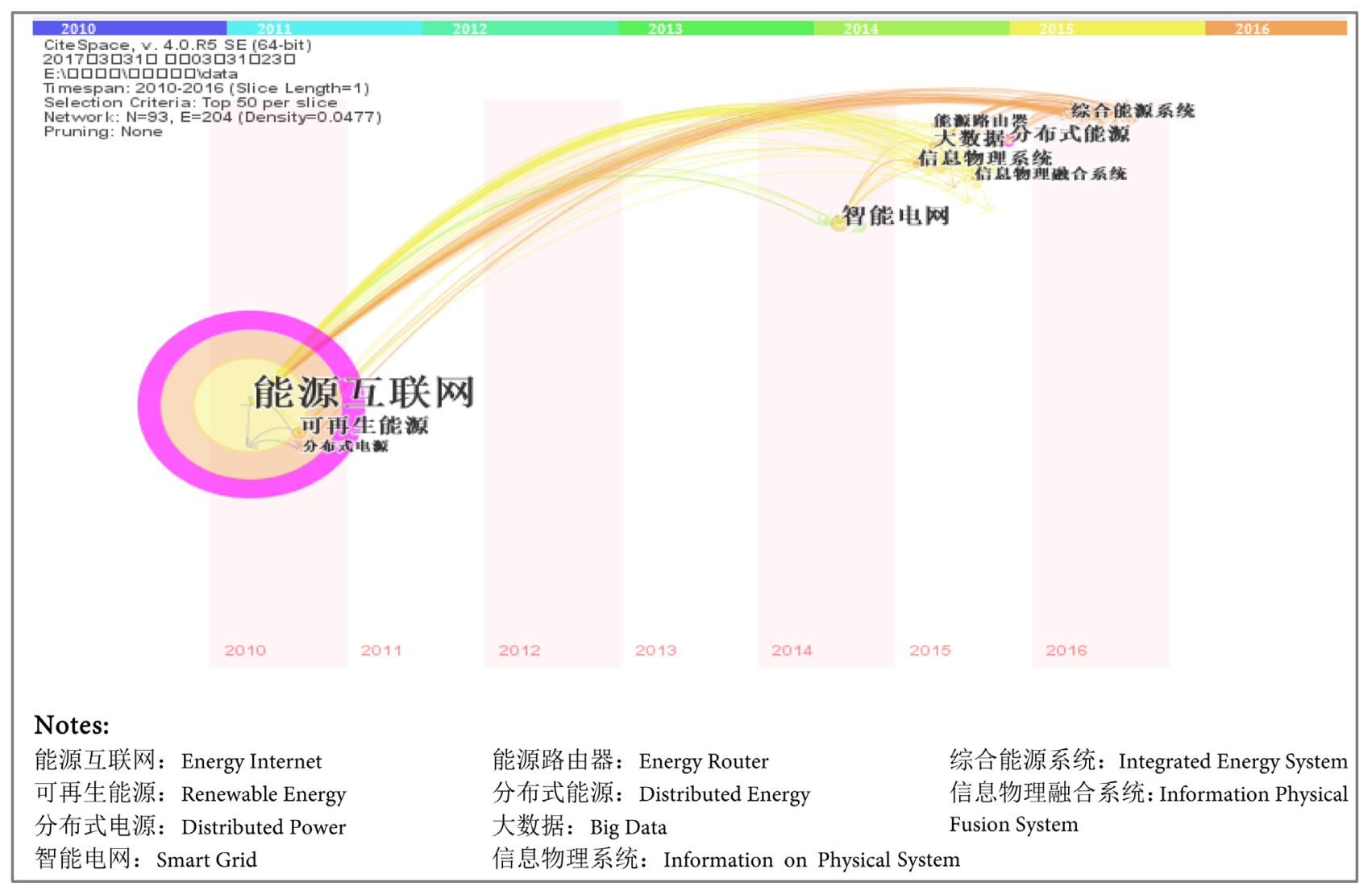

Figure 4. The time-zone view of keyword co-occurrence network.

Next, we'd like to explore the evolution of research hotspots. Therefore, we draw the time-zone view of keyword co-occurrence network. Figure 4 vividly shows the keyword evolution in domain of the energy internet between 2010 and 2016 in China. This concept was put forward for the first time in 2010; in 2014, the research topics changed to smart grid. In the recent period, main focuses have physical distributed energy, large data, energy router and integrated energy systems and so on. We can see that the energy internet got rapid development since it put forward in China.

\section{Conclusion}

In this study, we employed the informatics approach to discover the research hotspots and frontier technology of the smart power distribution and utilization domain, the hotspots focus on the power quality, distributed power supply and so on, and the energy internet and the active distribution network are the main development directions now and in the future. For the power quality and the energy internet, we drew the visualization graph of the keyword co-occurrence network through CiteSpace based on the methods of keyword co-occurrence analysis and cluster analysis.

\section{Acknowledgements}

The work was supported by Science Project of State Grid Corporation of China 
(52094015001L).

\section{References}

[1] China Electricity Council. (2016) Research Report on Standard System of Power Distribution and Utilization in the "13th Five-Year" Period. http://sanwen.net/a/yuqbzbo.html

[2] Wang, C.S., Wang, S.X.andGuo, L. (2010)Prospect over the techniques of smart distribution network in China. Southern Power System Technology, 4, 18-22.

[3] Wang, C.S., Wang, D.and Zhou, Y. (2015)Framework analysis and technical challenges to smart distribution system.AutomElectr Power Syst, 39, 2-9.

[4] Wang, B.B., Zhao, S.N. and Liu, X.C. (2016)Review on Key Technologies of Smart Power Utilization for Renewable Energy Integration, Power System Technology. 40, 3894-3903.

[5] Ma,Z.,An,T. and Shang, Y.W. (2016)State of the Art and Development Trends of Power Distribution Technologies. Proceedings of the CSEE. 36, 1552-1567.

[6] Wang,J.Y., Ji,Z.X. and Shi, M.J. (2015)Scenario Analysis and Application Research on Big Data in Smart Power Distribution and Consumption Systems.Proceedings of the CSEE. 35, 1829-1836.

[7] Li,W.L.,Tian,W. and Wang, .X.D. (2011)Research of Power Distribution Technology System Centering on AMI. Jiangsu Electrical Engineering,30, 1-6.

[8] Liu, T.Y. (1994)The Several Basic Research Problems in Informetrics. Information Science. 15, 55-64.

[9] Lee, J.Y. and Choi, S. (2010)Intellectual Structure and Infrastructure of Informetrics: Domain Analysis from 2001 to 2010. Journal of the Korean Society for Information Management.28, 11-36.https://doi.org/10.9708/jksci.2010.15.10.011

[10] Zhao,R.Y., Liu, H.H. and Wang, H.X. (2009)A Bibliometrics Study on Research Competitiveness of Engineering Universities in China, Management and Service Science, 2009. MASS'09. International Conference on. IEEE,Xiamen, 1-4.

[11] Zeng,J. X. (2016)Informatics Reform in Big Data http://www.1xuezhe.com/academic/detail?nid=224308\&tabtype=2

[12] Li,J. andChen, C.(2016) CiteSpace: Text Ming and Visualization in Scientific Literature. First Edition. Capital University of Economics Business Press, Beijing.

[13] Chen,Y., Chen,C. and Wang, Z.Y. (2015) The Methodology Function of CiteSpace Mapping Knowledge Domains. Studies in Science of Science. 33, 242-253.

[14] Zhang,Q., Wang,Q.and Hao, J. X. (2016) Mapping Smart Tourism Research in China: A Semantic and Social Network Analysis using CiteSpace. Service Systems and Service Management (ICSSSM), 2016 13th International Conference on.IEEE. Kunming, 1-5.https://doi.org/10.1109/icsssm.2016.7538622

[15] Chen,Y., Chen,C. and Hu,Z.G. (2014) Principles and Applications of Analyzing a Citation Space. Science Press, Beijing. 
Submit or recommend next manuscript to SCIRP and we will provide best service for you:

Accepting pre-submission inquiries through Email, Facebook, LinkedIn, Twitter, etc. A wide selection of journals (inclusive of 9 subjects, more than 200 journals)

Providing 24-hour high-quality service

User-friendly online submission system

Fair and swift peer-review system

Efficient typesetting and proofreading procedure

Display of the result of downloads and visits, as well as the number of cited articles Maximum dissemination of your research work

Submit your manuscript at: http://papersubmission.scirp.org/

Or contact epe@scirp.org 